APAE Memó No. 183 WASFP 15

\title{
ATBAC CHECK CALCULATION ON THE IBM - 650 PROGRAM NO. 303
}




\section{DISCLAIMER}

This report was prepared as an account of work sponsored by an agency of the United States Government. Neither the United States Government nor any agency Thereof, nor any of their employees, makes any warranty, express or implied, or assumes any legal liability or responsibility for the accuracy, completeness, or usefulness of any information, apparatus, product, or process disclosed, or represents that its use would not infringe privately owned rights. Reference herein to any specific commercial product, process, or service by trade name, trademark, manufacturer, or otherwise does not necessarily constitute or imply its endorsement, recommendation, or favoring by the United States Government or any agency thereof. The views and opinions of authors expressed herein do not necessarily state or reflect those of the United States Government or any agency thereof. 


\section{DISCLAIMER}

Portions of this document may be illegible in electronic image products. Images are produced from the best available original document. 


\section{ATBAC CHECK CALCULATION \\ ON THE IBM 650 \\ PROGRAM NO. 303}

\section{AEC CONTRACT NO. AT $(30-3)-326$}

\section{Issued March 13, 1959}

$$
\begin{gathered}
\text { I. Beretsky } \\
\text { P.V. Oby }
\end{gathered}
$$

Alco Products, Inc. Post Office Box 414 Schenectady $5, \mathrm{~N} . \mathrm{I}$. 


\section{DISTRIBUTION}

Copies

$1-2$

New York Operations Office

U. S. Atomic Energy Commission

70 Columbus Avenue

New York 23, New York

Attention: Capt. Richard L. Harris

(V.J. Del Vecchia for C)

$3-4$

U. S. Atomic Energy Commission

Washington 25, D。C.

Attention: Classified Technical Library for

Col. D.G. Williams

$5-7$

U. S. Atomic Energy Commission

Army Reactors Branch

Division of Reactor Development

Washington 25, D.C.

Attention: Major Paul H. Ugis

8

U. S. Atomic Energy Commission

Chief, Patents Branch

Washington 25, D.C.

Attention: Roland A. Anderson

9

U. S. Atomic Energy Commission

Chicago Operations Office

P. 0. Box 59

Lamont, Illinois

Attention: Captain J. Schweizer

10

U. S. Atomic Energy Commission

Idaho Operations Office

Phillips Petroleum Company, NRTS

Technical Library

P. O. Box 1250

Idaho Falls, Idaho

Attention: Major Robert L. Ednie

11

Nuclear Power Field Office

USERDL

Fort Belvoir, Virginia

Attention: Major W. R. Wray 


\section{DISTRIBUTION (Con't)}

Copies

12

Union Carbide Nuclear Corporation

Oak Ridge National Laboratory

Y-12 Building 9704-1

P. 0. Box "Y"

Oak Ridge, Tennessee

Attention: "A. L. Boch

13

District Engineer, Alaska District

U. S: Army Corps of Engineers

P. 0. Box 7002

Anchorage, Alaska

Attention: NPAVG $-\mathrm{N}$

14

The Martin Company

P. 0. Box 5042

Middle River, Maryland

Attention: AEC Contract Document. Custodian

$15-39$

U. S. Atomic Energy Commission

Reference Branch

Technical Information Services Extension

P. 0. Box 62

Oak Ridge, Tennessee

$40-41$

Alco Products, Inc。

P. 0. Box 145

Fort Belvoir, Virginia

Attention: H. L. Weinberg

42
43
44
45
46
47
48
$49-50$
$51-53$
$54-58$

K. Kasschau

J.F. Haines

W.M.S. Richards

J.G. Gallagher

$J . D$. Love

J.0: Brondel

F.T. Matthews

I. Beretsky

P.V. Oby

File 


\section{ABSTRACT}

I INTRODUCTION

II EQUATIONS $\quad 2$

III PROGRAM INSTRUCTIONS 3

A. Calculation Flow 3

B. Input Format 4

1. Floating Point 4

2. Fixed Point 4

C. Output Format 4

D. Machine Operation 4

1. Deck 4

2. Console Settings 4

3. Control Panel 4

E. Flow Chart 11

IV NOMENCLATURE $\quad 5$

TABLES

1 Input Format 8

2 Output Format $\quad 10$ 


\section{ABSTRACT}

This program, using the IBM 650 electronic data processing machine is used primarily as a tool in order to prepare input data for an IBM 704 code, entitled ATBAC. The code calculates steady state thermal characteristics of a plate type fuel element for both a nominal and adverse channel. 


\section{INTRODUCTION}

The purpose of this work is to present a tool to be used in conjunction with the ATBAC Code (WAPD $-T M-20$ ) in predicting the steady state temperature profiles in both nominal and adverse channels.

The principle quantities involved in this analysis are coolant flow rates, heat transfer coefficients, and local and average heat fluxes.

The nominal case offers an approximate indication of reactor conditions as they would be if most of the uncertainties were eliminated. In addition, it offers a stable "base" for the design factors which frequently change as the design of the core progresses. However, definition of the nominal case is somewhat arbitrary.

This report shows how the IBM 650 calculates both adverse and nominal temperature distributions, for either one or two pass cores in any flow direction combination with the assumption of perfect mixing. 
II EQUATIONS SOLVED

$$
\begin{aligned}
& \text { 1.: } W_{n 1}=\frac{W_{T i}}{n_{f}-I_{1}} \\
& \text { 2. } G_{n i}=\frac{W_{n i}}{d_{n}-Y_{n}} \times 144 \\
& \text { 3. } q_{a v}=\frac{2 q_{a v}^{n} s_{f} H_{p}}{144}
\end{aligned}
$$

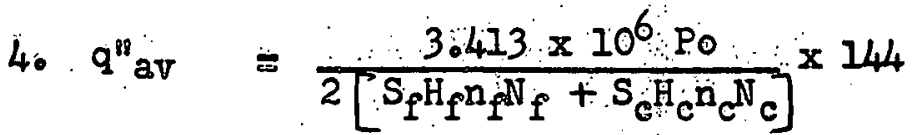

$$
\begin{aligned}
& \text { 5. } F_{\text {weng }}=\left(\frac{d_{\text {minav }}}{d_{n}}\right)^{5} \times \frac{Y_{\text {minav }}}{Y_{n}} \times\left(\frac{L_{\text {nom }}}{I_{\max }}\right)^{\frac{5}{9}} \\
& \text { 6. } F_{\text {heng }}=\frac{\left(d_{\text {minav }}\right) \frac{4}{3}}{d_{\max }\left(d_{n}\right) \cdot \frac{1}{3}} \times\left(\frac{I_{\text {mom }}}{I_{\max }}\right)^{\frac{4}{9}} \times\left(\frac{Y_{\operatorname{minav}}}{Y_{\max }}\right)^{\frac{4}{5}} \\
& \text { 7. } h_{n i}=K_{i} \cdot \frac{\left(G_{n i}\right)^{0.8}}{\left(d_{n} / 6\right)^{0.2}}
\end{aligned}
$$$$
\text { 8. } W_{\min }=W_{\mathrm{ni}} \times F_{\text {weng }} \times \mathrm{F}_{\mathrm{pl}}
$$$$
\text { 9. } G_{\min i}=\frac{W_{\operatorname{mini}}}{d_{\max } Y_{\max }} \times 144
$$$$
\text { 10. } h_{\text {mini }}=h_{n i} \times F_{\text {heng }} \times\left(F_{p l}\right) \frac{4}{5} \times F_{h}
$$$$
\text { 11. } h_{\text {avmini }}=\left(\frac{d_{\text {minav }}}{d_{n}}\right)^{\frac{1}{3}} \times F_{h} \times h_{n i}
$$

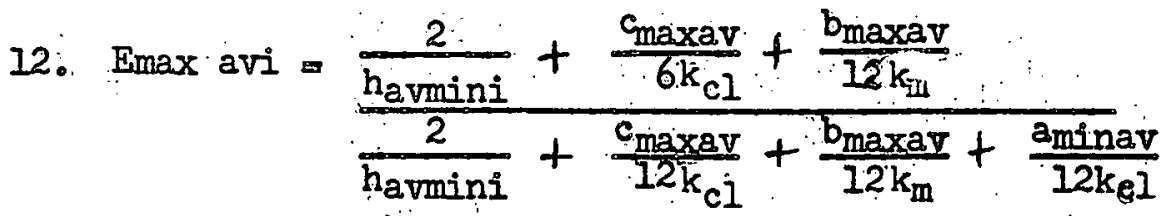$$
\text { 13. } E_{\operatorname{maxloci}}=\frac{\frac{2}{h_{\operatorname{mini}}}+\frac{c_{\operatorname{maxl} 0 c}}{6 k_{\mathrm{cl}}}+\frac{\mathrm{b}_{\operatorname{maxloc}}}{12 \mathrm{k}_{\mathrm{m}}}}{\frac{2}{h_{\min l 1}}+\frac{c_{\operatorname{maxlloc}}}{12 \mathrm{k}_{\mathrm{cl}}}+\frac{b_{\operatorname{maxloc}}}{12 \mathrm{k}_{\mathrm{m}}}+\frac{a_{\operatorname{minloc}}}{12 \mathrm{k}_{\mathrm{cl}}}}
$$$$
\text { 14. } F_{\text {qengi }}=E_{\text {maxavi }} \times \frac{b_{\text {maxav }}}{b_{n o m}} \times \frac{D_{\text {maxav }}}{D_{n}} \times \frac{S_{\text {maxav }}}{S_{f}}
$$ 
II EQUATIONS SOLVED (Cont)

15. $F_{\text {q"engi }}=E_{\operatorname{maxloci}} \times \frac{\mathrm{b}_{\max } \mathrm{b}_{\text {noc }}}{b_{\text {nom }}} \times \frac{\mathrm{D}_{\operatorname{maxloc}}}{D_{\mathrm{n}}}$

16. $R e_{\min }=\frac{d_{n} G_{n i}}{6 \mu}$

17. $R e_{\max }=\frac{d_{\operatorname{minav}} G_{\operatorname{mini}}}{6 \mu}$

18. $\mathrm{T}_{\text {wni }}(\mathrm{Z})=\mathrm{T}_{\text {in-nomi }}+\frac{q_{\mathrm{av}} \mathrm{F}_{\mathrm{cpi}}(\mathrm{Z})}{\mathrm{W}_{\mathrm{ni}} \mathrm{C}_{\mathrm{pi}}}$

19. $T_{\text {sni }}(z)=T_{w n i}(z)+\frac{q^{\prime \prime} v^{F_{r}{ }_{i j}}(z)}{h_{n i}}$

20. $\mathrm{T}_{\text {wmaxi }}(\mathrm{Z})=\mathrm{T}_{\text {inmaxi }}+\frac{\mathrm{q}_{\mathrm{av}} \mathrm{F}_{\text {cppi }}(\mathrm{Z}) \mathrm{F}_{\mathrm{qengi}}}{\mathrm{W}_{\operatorname{mini}} \mathrm{C}_{\mathrm{pi}}}$

21. $\mathrm{T}_{\text {Smaxi }}(\mathrm{Z})=\mathrm{T}_{\text {wmaxi }}(\mathrm{Z})+\frac{\mathrm{q}^{\prime \prime}{ }_{\text {av }} \mathrm{F}_{\mathrm{rlpi}}(\mathrm{Z}) \mathrm{F}_{\mathrm{q}^{\prime \prime} \text { engi }}}{\mathrm{h}_{\operatorname{mini}}}$

22. $\mathrm{T}_{\text {in nom2 }}=\mathrm{T}_{\text {in } \max 2}=\mathrm{T}_{\mathrm{wni}}(\mathrm{Z}=\mathrm{I})$

\section{PROGRAM INSTRUCTIONS}

A. Calculation Flow (see flow chart)

1. Equations 1 through 17 are solved for the first pass $(i=1$ ).

2. Results thus far are punched out.

3. Equations 18 through 21 are solved and results are punched out for $\mathrm{Z}=0$ through $\mathrm{L}$.

4. Equation 22 is satisfied and steps 1 through 3 are repeated for the second pass ( $\mathrm{Z}$ goes from $\mathrm{L}$ to 0 ).

5. After the 2nd pass (or Ist if only I pass), machine goes to read the next case input. 


\section{B. Input Format}

All input is entered into the 650 in 7 values/card Fortransit format. Forms are available for the input. All input except $I$ and $N$ are in floating point form.

1. Floating Point Form

$$
\begin{aligned}
& . \text { XXXXXXXXPP } \text { PP }=c c+50 \\
& \text { Example } 127.3=.1273 \times 10^{3} \longrightarrow 1273000053 \\
& .0876=.876 \times 10^{-1} \longrightarrow 8760000049 \\
&-.00294=-.294 \times 10^{-2} \rightarrow-2940000048
\end{aligned}
$$

2. Fixed Point Form

$. \times x \times x \times x \times x \times x$

Example $\quad 3 \rightarrow 0000000003$

$93276 \rightarrow 0000093276$

$-22 \rightarrow-0000000022$

C. Output Format

All output occurs three values per card with associated identi-

fication. All output is in floating point form except the number of pass and the $\mathrm{Z}$ index.

D. Machine Operation

1. Deck consists of:

a. Program deck

b. Input

2. Console settings:

7019529999 STOP, RUN, ANYTHING, RUN, UPPER, SENSE, STOP

3. Control Panel:

IT-SOAP 
IV NOMENCLATURE

$$
\begin{aligned}
& \mathrm{C}_{\mathrm{p}}=\text { Average specific heat, hot channel, } \mathrm{Btu} / \mathrm{Ib}{ }^{\circ} \mathrm{F} \\
& \overline{\mathrm{c}}_{\mathrm{p}}=\text { Average specific heat, nominal channel, Btu/IboF } \\
& \text { D = Relative uranium density } \\
& \text { E = Eccentricity Factor: Accounts for increased heat flux } \\
& \text { away from fuel element in one particular direction. } \\
& F_{h} \quad=\text { Film coefficient factor, accounts for inaccuracy in } \\
& \text { the correlation of } h \\
& F_{\text {pl }} \quad=\text { Plenum Factor: Accounts for the maldestribution of } \\
& \text { flow among fuel boxes and channels } \\
& F_{r l}(Z) \quad=\text { Average power at a particular level of a specified } \\
& \text { fuel element/Average power of reactor } \\
& F_{\text {rlp }}(Z) \quad=\text { Maximum power at a particular level of a specified } \\
& \text { fuel element/Average power of reactor } \\
& F_{\text {ep }}(Z)=\frac{I}{\bar{I}} \int_{0}^{Z} F_{r I}(Z) d Z \\
& \text { - Average cumulative power per unit length developed up } \\
& \text { to a particular level of a specified fuel element/ } \\
& \text { Average power of reactor. } \\
& F_{\text {cpp }}(Z)=\frac{I}{L} \int_{0}^{Z} F_{r l p}(Z) d Z \\
& \text { = Maximum cumulative power per unit length developed up } \\
& \text { to a particular level of a specified fuel element/ } \\
& \text { Average power of reactor } \\
& F_{\text {h-eng }} \quad=\text { Engineering factor applied to film coefficient }
\end{aligned}
$$


IV NOMENCLATURE (Con $t$ )

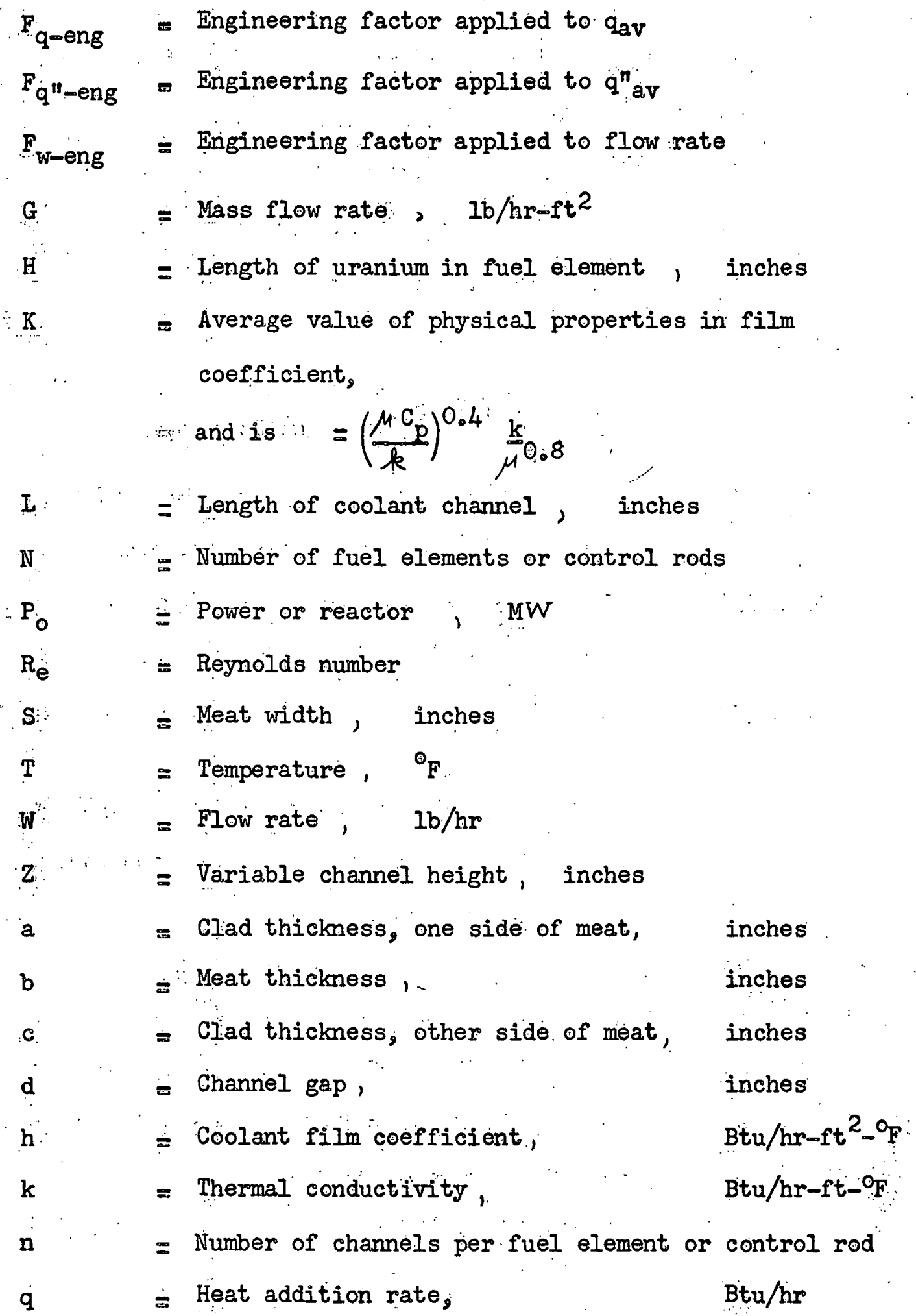


IV NOMENCLATURE (Con't)

$\begin{aligned} q^{\prime \prime} & =\text { Heat flux } \quad \text { Btu } / \mathrm{hr}^{\prime} \mathrm{ft}^{2} \\ p & =\text { Density of coolant Ib/ft }{ }^{3} \\ \mu & =\text { Viscosity of coolant } \mathrm{lb} / \mathrm{ft}-\mathrm{hr}\end{aligned}$

\section{Subscripts}

$\begin{array}{ll}\text { av } & =\text { Average } \\ \text { eng } & =\text { Based on engineering tolerances } \\ \text { loc } & =\text { Based on local extreme } \\ \max & =\text { Maximum (local) } \\ \min & =\text { Minimum (local) } \\ \mathrm{n} & =\text { Nominal } \\ \mathrm{w} & =\text { Bulk water } \\ \mathrm{S} & =\text { Surface } \\ \mathrm{i} & =\text { Pass number } \\ \mathrm{f} & =\text { Fuel element } \\ \mathrm{c} & =\text { Control rod } \\ \mathrm{T} & =\text { Total } \\ \mathrm{cl} & =\text { Cladding }\end{array}$


TABLE 1

INPUT FORMAT

\begin{tabular}{|c|c|c|c|c|c|c|c|}
\hline Word I & Word 2 & Word 3 & Word 4 & Word 5 & Word 6 & Word 7 & Word 8 \\
\hline 0202510007 & $a_{\min } a v$ & $b_{\text {nom }}$ & $b_{\max } a v$ & $b_{\max } l o c$ & $c_{\max a v}$ & $d_{n}$ & $\alpha_{\max }$ \\
\hline 0202580007 & $d_{\min } a v$ & $D_{\max }$ av & $P_{\max } 10 c$ & $D_{n}$ & $\mathrm{H}_{\mathrm{f}}$ & $\mathrm{H}_{\mathrm{c}}$ & $I_{\text {nom }}$ \\
\hline 0202650007 & $\mathrm{~L}_{\max }$ & $\mathrm{N}_{f}$ & $\mathrm{~N}_{\mathrm{c}}$ & $\mathrm{N}_{\mathrm{f}}$ & $\mathrm{N}_{\mathrm{c}}$ & $s_{f}$ & $\mathrm{~s}_{\mathrm{c}}$ \\
\hline 0202720007 & $S_{\max } a v$ & Tin nom & $F_{\text {in maxi }}$ & $Y_{n}$ & $Y_{\max }$ & $Y_{\min }$ av & $a_{\min } 10 c$ \\
\hline 0202790007 & $\mathbf{c}_{\max }$ av & Zeros & $P_{0}$ & $\mu$ & $\mathrm{k}_{\mathrm{c}}$ & $\mathrm{k}_{\mathrm{m}}$ & $F_{p l}$ \\
\hline 0202860007 & $F_{h}$ & $\mathrm{~K}_{1}$ & $\kappa_{2}$ & $c_{p l}$ & $c_{p 2}$ & $\overline{\mathrm{c}}_{\mathrm{pl}}$ & $\bar{c}_{p 2}$ \\
\hline 0200510007 & $F_{\text {cpl }}(0)$ & $F_{\mathrm{cp} 2}(0)$ & $F_{\mathrm{cpl}}\left(\mathrm{z}_{1}\right)$ & $F_{\mathrm{cp} 2}\left(\mathrm{z}_{1}\right)$ & $\mathrm{F}_{\mathrm{cpl}}\left(\mathrm{z}_{2}\right)$ & $\mathrm{F}_{\mathrm{cp2}}\left(\mathrm{z}_{2}\right)$ & $F_{c p l}\left(z_{3}\right)$ \\
\hline 0200580007 & $\mathrm{~F}_{\mathrm{cp} 2}\left(\mathrm{z}_{3}\right)$ & etc. for & all $\mathrm{z}$ vali & & & & \\
\hline 0201010007 & $F_{\text {rl }}$ valu & $s$ in same & fashion as & $\mathrm{F}_{\mathrm{cp}}$ & & & \\
\hline 0201510007 & $F_{\text {cpp }}$ val & es in sam & e fashion as & $F_{c p}$ & & & \\
\hline 0202010007 & $F_{\text {rlp }}$ val & des in same & e fashion as & $F_{c p}$ & & & \\
\hline 0100040002 & No. $z$ va & Lues No. pe & asses & & & & \\
\hline 0202930002 & $w_{T_{1}}$ & $\mathrm{~W}_{\mathrm{T} 2}$ & & & & & \\
\hline
\end{tabular}


1. As many as 8 cards may be used for the $F_{\mathrm{cp}}$ array. Similarly for $F_{\mathrm{rl}}, F_{\mathrm{cpp}}$, and $F_{\mathrm{rlp}}$. Maximum number of values of each is 25/pass. On the last card of each set, the number 7 should be replaced with the number of values on the card. Values 0051, 0058, 0101, 0151,0201 index by 7.

2. The maximum number of $\mathrm{Z}$ values is 25. The first value should be for $\mathrm{Z}=0$.

3. After a calculation is complete, additional values of $W_{T} l$ and $W_{T}$ ? may be read into the 650 for additional calculation.

4. For a completely new calculation, the program deck must be read in after resetting the computer. 
OUTPUT FORMAT

\begin{tabular}{|c|c|c|c|c|c|}
\hline Word 1 & Word 2 & Word 3 & Word 4 & Word 5 & Word 6 \\
\hline $0200070008=$ & q"av & 0200080008 & qav & 0200110008 & $F_{\text {weng }}$ \\
\hline $0200130008-$ & $F_{\text {heng }}$ & & & & \\
\hline 0202930010 & nTI $_{\mathrm{T}}$ & 0201940010 & $\mathrm{~W}_{\mathrm{T} 2}$ & 0100020010 & Pass No. \\
\hline $0200030010-$ & $w_{n i}$ & 0200040010 & $G_{n i}$ & 0200140010 & $h_{n i}$ \\
\hline $0200150010=$ & $w_{\min i}$ & 0200160010 & $G_{\text {mini }}$ & 0200170010 & $\mathrm{~h}_{\operatorname{mini}}$ \\
\hline 0200180010 & $h_{a v \operatorname{mini}}$ & 0200210010 & $F_{\text {ceng }}$ & 0200220010 & Fq"eng \\
\hline $0200230010-$ & $\mathrm{Re}_{\min }$ & 0200240010 & $\mathrm{Re}_{\max }$ & & \\
\hline $0100030017-$ & Z\# & 0200250017 & $\mathrm{~T}_{\mathrm{wni}}$ & 0200260017 & $\mathrm{~T}_{\text {sni }}$ \\
\hline $0100030017-$ & Z\# & 0200270017 & $\mathrm{~T}_{\text {Wmaxi }}$ & 0200280017 & $\mathrm{~T}_{\text {Smaxi }}$ \\
\hline $0100030017-$ & Z\# & 0200250017 & $\mathrm{~T}_{\text {wni }}$ & 0200260017 & $\mathrm{~T}_{\mathrm{sni}}$ \\
\hline
\end{tabular}

Z\# begins with 1 for the $Z=0$ value

The above output is repeated for the second pass if a second pass is specified 


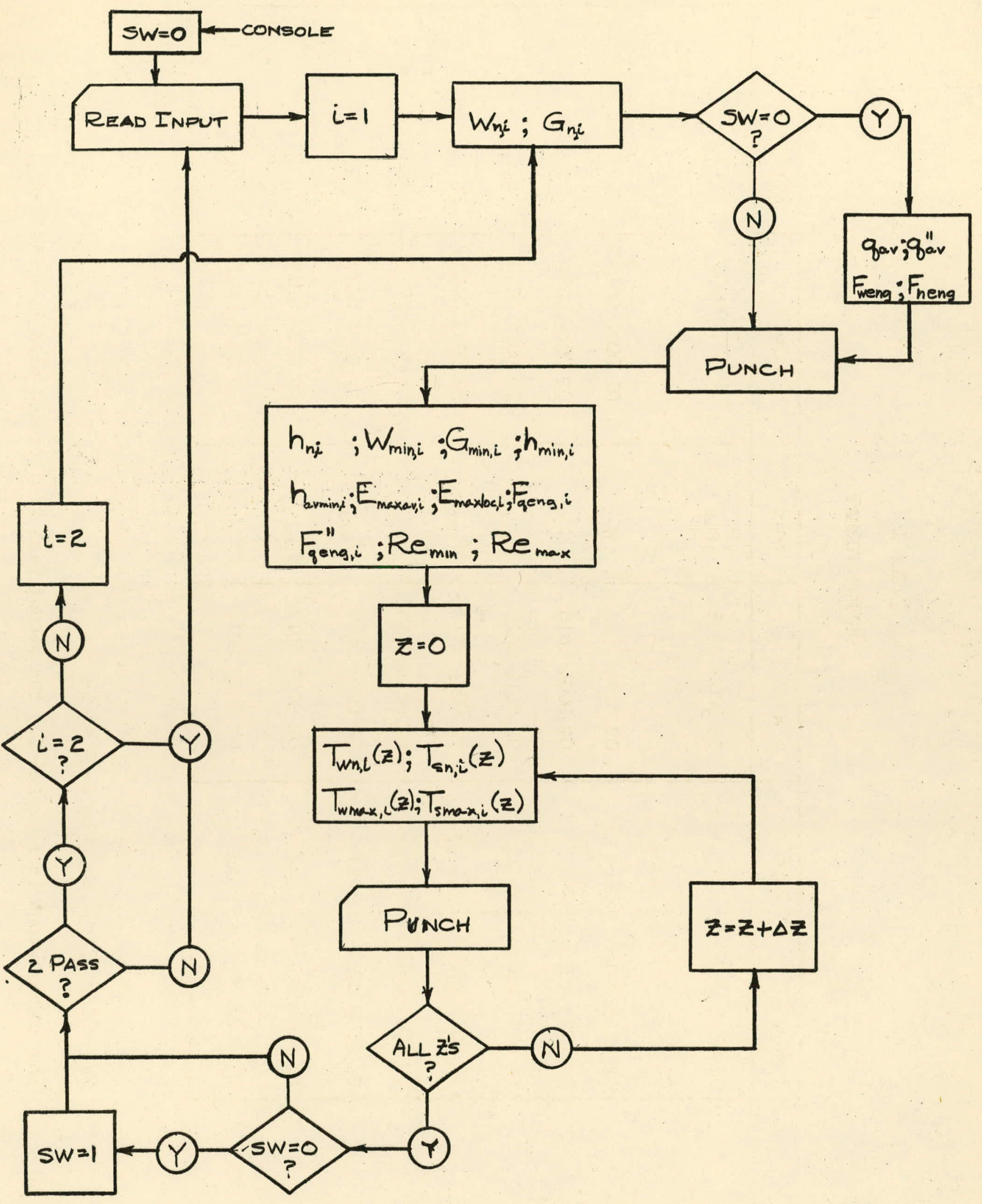

